\title{
$\begin{array}{r}\text { WAGENINGEN } \\ \text { UNIVERSITY \& RESEARCH } \\ \hline\end{array}$
}

\section{VIS/NIR imaging application for honey floral origin determination}

Minaei, S., Shafiee, S., Polder, G., Moghadam-Charkari, N., van Ruth, S., Barzegar, M., ... Kuś, P. M.

This is a "Post-Print" accepted manuscript, which has been published in "Infrared Physics \& Technology"

This version is distributed under a non-commercial no derivatives Creative Commons (C) $(1) \Theta($ (CC-BY-NC-ND) user license, which permits use, distribution, and reproduction in any medium, provided the original work is properly cited and not used for commercial purposes. Further, the restriction applies that if you remix, transform, or build upon the material, you may not distribute the modified material.

Please cite this publication as follows:

Minaei, S., Shafiee, S., Polder, G., Moghadam-Charkari, N., van Ruth, S., Barzegar, M., ... Kuś, P. M. (2017). VIS/NIR imaging application for honey floral origin determination. Infrared Physics \& Technology, 86, 218-225. DOI:

10.1016/j.infrared.2017.09.001

You can download the published version at:

https://doi.org/10.1016/j.infrared.2017.09.001 


\section{Accepted Manuscript}

VIS/NIR imaging application for honey floral origin determination

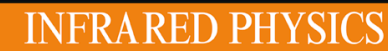

INFRARED PHYSICS

VIS/NIR imaging application for honey floral origin determination

Saeid Minaei, Sahameh Shafiee, Gerrit Polder, Nasrolah Moghadam-Charkari, Saskia van Ruth, Mohsen Barzegar, Javad Zahiri, Martin Alewijn, Piotr M.

Kuś

PII:

S1350-4495(17)30261-X

DOI: http://dx.doi.org/10.1016/j.infrared.2017.09.001

Reference:

INFPHY 2372

To appear in: $\quad$ Infrared Physics \& Technology

Received Date: $\quad 11$ May 2017

Revised Date: $\quad 31$ August 2017

Accepted Date: $\quad 3$ September 2017

Please cite this article as: S. Minaei, S. Shafiee, G. Polder, N. Moghadam-Charkari, S. van Ruth, M. Barzegar, J. Zahiri, M. Alewijn, P.M. Kuś, VIS/NIR imaging application for honey floral origin determination, Infrared Physics \& Technology (2017), doi: http://dx.doi.org/10.1016/j.infrared.2017.09.001

This is a PDF file of an unedited manuscript that has been accepted for publication. As a service to our customers we are providing this early version of the manuscript. The manuscript will undergo copyediting, typesetting, and review of the resulting proof before it is published in its final form. Please note that during the production process errors may be discovered which could affect the content, and all legal disclaimers that apply to the journal pertain. 
Research

Title

\title{
VIS/NIR Imaging Application for Honey Floral Origin Determination
}

Authors

Saeid Minaei ${ }^{\mathrm{a}}$, Sahameh Shafiee ${ }^{\mathrm{a} *}$, Gerrit Polder $^{\mathrm{b}}$, Nasrolah Moghadam-Charkari ${ }^{\mathrm{c}}$, Sáskia van Ruth $^{\text {de }}$, Mohsen Barzegar ${ }^{\mathrm{f}}$, Javad Zahiri ${ }^{\mathrm{g}}$, Martin Alewijn ${ }^{\mathrm{e}}$, Piotr M. Kuś ${ }^{\mathrm{dh}}$

${ }^{a}$ Biosystems Engineering Department, Tarbiat Modares University, Tehran, Iran

${ }^{\text {b}}$ Wageningen University \& Research, Greenhouse Horticulture, Wageningen, Netherlands

${ }^{\mathrm{c}}$ Department of Computer science, Tarbiat Modares University, Tehran, Iran

${ }^{\mathrm{d}}$ RIKILT Wageningen University \& Research, Wageningen, the Netherlands

${ }^{\mathrm{e}}$ Wageningen University\& Research, Food Quality and Design Group, Wageningen, the Netherlands

${ }^{\mathrm{f}}$ Department of Food Science and Technology, Tarbiat Modares University, Tehran, Iran

g Bioinformatics and Computational Omics Lab (BioCOOL), Department of Biophysics, Tarbiat Modares University, Tehran, Iran

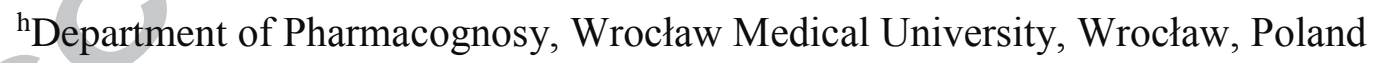

\author{
Corresponding author \\ shafie@isipo.ir
}

Tel:+98-2148292466

Fax: +98-2148292200 


\begin{abstract}
Nondestructive methods are of utmost importance for honey characterization. This study investigates the potential application of VIS-NIR hyperspectral imaging for detection of honey flower origin detection using machine learning techniques. Hyperspectral images of 52 honey samples were taken in transmittance mode in the visible/near infrared (VIS-NIR) range (400-1000 nm). Three different machine learning algorithms were implemented to predict honey floral origin using honey spectral images. These methods, included radial basis function (RBF) network, support vector machine (SVM), and random forest (RF). Principal component analysis (PCA) was also exploited for dimensionality reduction. According to the obtained results, the best classifier (RBF) achieved a precision of $94 \%$ in a five-fold cross validation experiment using only the first two PCs. Mapping of the classifier results to the test set images showed $90 \%$ accuracy for honey images. Three types of honey including buckwheat, rapeseed and heather were classified with $100 \%$ accuracy. The proposed approach has great potential for honey floral origin detection. As some other honey properties can also be predicted using image features, in addition to floral origin detection, this method may be applied to predict other honey characteristics.
\end{abstract}

Keywords: Honey Floral origin, NIR Hyperspectral imaging, Random forest, Radial basis function network, Support vector machine.

\title{
1. Introduction
}

With globalization of the honey market, which involves approximately 150 countries, identification of honey origin as well as the proof of its authenticity has become an important issue [1]. Demand for verification of honey floral origin has increased in the past few years, because of the high consumer preference for specific types of honey due to their differently sensory perceived and medicinal properties. Determination of the floral origin of honey is important from an economic point of view since monofloral honeys have higher commercial 
value than polyfloral ones [2]. Therefore, the search for reliable methods indicating honey floral origin has been the focus of many studies [1].

Traditionally, analysis of honey pollen content or "melissopalynology" has been the most prevalent technique to classify different honey types. Some disadvantages of this method such as alteration of pollen content in honey by the action of bees or adulteration by the action of fraudsters have been reported [3]. Other shortcomings include: removal of pollen in the filtration process [4], inability to identify the source of some special honeys such as cotton, castor-oil plants, and rubber tree [3], as well as the difficulty in differentiating between various types of honey having similar pollen grains [5]. Furthermore, this method is not suitable for rapid routine analysis [6-7].

The honey industry needs simple and fast methods (for routine analysis of numerous samples in a short period) for determination of honey flower origin. Thus, many studies have focused on the utilization of instrumental techniques allowing such analyses. Evaluation of some physical or chemical properties and sensory characteristics of honey have been utilized to determine floral origin [8-9]. Researchers have attempted to characterize various honey samples by phytochemical floral markers such as volatile compounds, phenolic compounds, carbohydrates and nitrogen containing compounds [1]. Some methods require sample preparation and are destructive, for example instrumental methods such as: atomic absorption spectroscopy (AAS) [10], high-performance liquid chromatography [11], gas chromatography coupled with mass spectrometry (GC-MS) [12-13], electro-spray mass spectrometry (ES-MS) [14], inductively-coupled plasma optical emission spectrometry (ICPOES) [7], thin-layer chromatography (TLC) [15], and high-performance anion-exchange chromatography with pulsed amperometric detection (HPAED-PAD) [16]. Different spectroscopy techniques such as, nuclear magnetic resonance (NMR) [17-18], Fourier transform-Raman (FT-Raman) [19], Fourier- transform infrared (FTIR) [20], mid-infrared 
spectroscopy [21], and near-infrared (NIR) spectroscopy [21-24] have been utilized by various researchers. NIR spectroscopy is considered as a fast and non-destructive method for determination of honey floral origin among different spectroscopic techniques [24]. Machine vision has been widely used as a non-destructive and fast method for food characterization. In the case of honey, this method was proposed as an effective approach for honey characterization by Shafiee et al (2013) [25]. In addition, prediction of some nutrients in honey was studied using RGB images [26]. To establish relationships between honey color and its nutrient content, authors have proposed the use of RGB images for honey characterization. However, this method would not be effective in differentiating honey samples having the same color [27]. Since, the intensity values of RGB images are measured in three channels, increasing the number of imaging bands can lead to an extension of the extracted information from an image. Hyperspectral images normally contain information in different bands with different resolutions [28]. Recently, authors applied hyperspectral imaging system successfully to honey adulteration detection [29]. The common definition for hyperspectral imaging is the acquisition of spatial images in many spectrally continuous bands measured from a remotely operated platform [30]. The basic principle of hyperspectral imaging is founded on the fact that all materials, due to the differences in their chemical composition and inherent physical structure, reflect, scatter, absorb, and emit electromagnetic energy in distinctive patterns at specific wavelengths. This characteristic is called spectral signature or spectral fingerprint, or simply the spectrum [31]. Spectral imaging allows the measurement of spatial and spectral information simultaneously that would not be measured with regular spectroscopic measurements or common imaging methods. Thus, it integrates the main advantages of spectroscopy and imaging for the quality prediction of food and agricultural products [28]. In essence, the spectral signature can be used to uniquely characterize, identify, and discriminate classes in each pixel of the image [32]. Various 
studies have been performed to show the ability and use of spectral imaging for food characterization including: meat and fish (beef tenderness prediction [33], pork quality and marbling level assessment [34], fat, color and moisture distribution in salmon fillets [35-37]); fruits and vegetables (citrus canker detection [38], internal quality of blueberries [39], tomato ripeness measurement [40], and discrimination of biological contaminants in fresh-cut lettuce [41]); as well as milk and dairy products [42]). An advantage of this technique is that in most cases no sample preparation is required prior to measurement. The fact that no chemical solvents are used is an advantage over chemical methods from an environmental point of view. In addition to the generation of a mean spectrum (the same as spectroscopy), spectral imaging is also able to store a volume spectrum for each point of the tested sample.

The purpose of this study is to develop a methodology to discriminate different types of unifloral honey (including: acacia, rapeseed, lime, buckwheat, and heather honey) using VISNIR hyperspectral imaging system in using machine learning techniques as a fast and nondestructive assessment tool.

\section{Materials and methods}

\subsection{Honey samples}

A total of 52 honey samples were used in this study including: 8 black locust (acacia) (Robinia pseudoacacia L.), 10 buckwheat (Fagopyrum esculentum Moench), 9 heather (Calluna vulgaris (L.) Hull), 15 lime (Tilia spp.), and 10 rapeseed (Brassica napus L.) samples. All the honey samples were collected in Poland and their floral origin was confirmed using pollen analysis. After receipt, the samples were stored at $4^{\circ} \mathrm{C}$ in dark environment. Qualitative and quantitative melissopalynological analyses were carried out in conformance with the method of the International Commission of Bee Botany and the International Honey Commission [43-44]. Honey samples were transferred onto microtiter plates having 12 sample wells which allow for simultaneous measurement of 12 samples in 
one image acquisition. They were placed in warm water bath overnight at $40^{\circ} \mathrm{C}$ to dissolve any crystalized sugars. A total of 20 samples (including 4 samples from each group) were kept out as the test set.

\subsection{VIS-NIR Image acquisition and processing}

Configuration of the VIS-NIR hyperspectral image acquisition set up is given in Fig.1. The system was calibrated prior to initial use [45]. Images were taken using a push-broom type line-scan camera consisting of a line scan spectrograph (Specim V10e) and a CMOS camera (PhotonFocus MV1, Finland). Due to the reflective nature of honey, image acquisition in reflectance mode produces considerable reflection from the surface of the samples. Thus, among different modes of hyperspectral imaging (namely: reflectance, transmittance, and translucence), transmittance mode was used as the sensing method for VIS-NIR imaging of honey samples. This mode involves minimum reflection from the honey surface. The light source and light tent are located underneath the sample.

The detector on the opposite side captures the light transmitted through the sample. In order to prevent specular reflection directly entering the detector, black sheets were utilized to cover the surface of the light diffuser surrounding the honey sample. A halogen-tungsten lamp was used as a broadband illumination source for visible and near-infrared spectral regions. A diffuser chamber was placed on top of the lamp to diffuse and scatter the light in different directions. Honey samples were placed on the top center of the diffuser and the camera on the opposite side. A stepper motor with a user-defined speed was utilized to move the camera on top of the honey sample. The camera and other parts of the VIS-NIR imaging system were placed on or attached to an aluminum frame $(700 \times 800 \times 900 \mathrm{~mm})$ as shown in Fig.1.

Image acquisition software (Isaac 2, custom-made software for hyperspectral data acquisition and visualization, Wageningen University \& Research, 2014) was utilized to control the 
different parts of the system to acquire images. The camera was moved over the honey samples scanning them line-by-line. Spectral images were collected in the spectral wavelength range of 400-1000nm with a resolution of $3.13 \mathrm{~nm}$ resulting in 192 spectral bands. The final dimension of each hyperspectral image is $100 \times 656$ pixels $\times 192$ spectral bands, where the first two numbers designate spatial dimensions ( $\mathrm{x}, \mathrm{y})$ and the third dimension stands for the number of spectral bands. This 3D data cube was saved as a folder with "tiff" images for each individual band along with meta information in xml format for further processing. In order to render the images independently of the spectral power distribution of the light source and the spectral sensitivity of the sensor, raw images were corrected using the following equation:

$$
\mathrm{R}=\frac{\mathrm{I}-\mathrm{B}}{\mathrm{W}-\mathrm{B}}
$$

Where $R$ is the corrected spectral image, $I$ is the raw spectral image, $W$ and $B$ are white and black reference images, respectively. Because of utilizing the transmittance mode, the image of the background sheet was used as the white reference while the dark reference was acquired with the light source off and the camera lens completely covered with its opaque cap. The software implemented this correction before capturing each image.

\subsection{Pixel selection and spectral information pre-processing}

An image of the micro-titer plate containing honey samples was cropped from the background and extracted from each data cube. The images of 32 honey samples were implemented for pixel-base classification and 50 pixels were randomly chosen from a dataset collection of each honey group. Thus, for 5 different honey groups, 250 pixels were taken into consideration. Image background was expressed as a separate class such that 50 pixels were selected randomly for the background class. A total of 300 randomly selected pixels from six different classes were collected for spectral pixel-based classification. In the 
classification stage, the total set of 300 pixels, was divided into 5 bins and $70 \%$ of each bin was applied for training the classifiers and the remainder was used as the test set. This procedure was repeated 5 times (five-fold cross-validation) and the average performance is reported. The performance of the classifiers was assessed in terms of accuracy (ACC), precision (PRE), and recalls that are commonly stated as:

$\mathrm{ACC}=\frac{\mathrm{TP}+\mathrm{TN}}{\mathrm{TP}+\mathrm{FP}+\mathrm{TN}+\mathrm{FN}}$

$\mathrm{PRE}=\frac{\mathrm{TP}}{\mathrm{TP}+\mathrm{FP}}$

Recall $=\frac{\mathrm{TP}}{\mathrm{TP}+\mathrm{FN}}$

Where TP is the number of true positives, FP is the number of false positives, TN is the number of true negatives and $\mathrm{FN}$ is the number of false negatives [46]. In addition, the area under the receiver operating characteristic (ROC) curve was measured. In the ROC curve, a two-dimensional space is formulated using sensitivity as the vertical axis and specificity as the horizontal one. An area of 1 represents a perfect test; while, an area of 0.5 represents a worthless test. This means that we can select a robust model through maximizing the area under the ROC curve [47].

\subsection{Classification models}

Three classification methods including radial basis function (RBF) network, Support Vector Machine (SVM) and Random Forest (RF) were utilized to classify the spectral images.

Artificial neural network (ANN) is one of the most important pattern recognition methods which has well-established performance in hyperspectral image processing [48]. The RBF network is a special type of Artificial Neural Networks (ANN) with several distinctive features. A RBF network consists of three layers, including: the input layer, the hidden layer, and the output layer. The input layer broadcasts the coordinates of the input vector to each of 
the units in the hidden layer. Each unit in the hidden layer then produces an activation based on the associated radial basis function. Finally, each unit in the output layer computes a linear combination of the activations of the hidden units. How a RBF network reacts to a given input stimulus is completely determined by the activation functions associated with the hidden units and the weights associated with the links between the hidden layer and the output layer [49].

The support vector machine is a kernel-based machine learning algorithm. The basic idea of SVM is to find the optimal hyper plane as a decision surface that correctly separates the largest fraction of data points while maximizing the margins from the hyper plane to each class. As a kernel-based method, SVM appears to be especially advantageous in the analysis of hyperspectral data. SVM implements a maximum margin-based geological classification strategy, which shows the robustness of high dimensionality of the hyperspectral data and low sensitivity of the number of training data [48]. Different types of kernels such as polynomial, neural networks, and radial basis function (RBF) networks are used in SVM. This study uses the polynomial kernel for classifying different honey types.

Another classifier in this study is the Random Forest. This algorithm is a classification method consisting of many decision trees. In the training phase, each tree is constructed based on a subset of independent features. To classify a new object, put the input vector down each of the trees in the forest, and finally one class is assigned to the object based on majority voting [50]. Analyses were performed using a program written in Matlab 7 (The MathWorks Inc, MA, 2012a, USA).

\section{Results and discussion}

\subsection{Hyperspectral image classification}


A sample of the selected pixels and their related raw spectra are given in Fig.2, which shows the differences between the background and the honey samples. The blue spectrum belong to the honey samples whereas the red one is associated with the background.

Due to the use of the transmittance mode for spectral image acquisition, there are some shadows around each well of the microtiter plate. In these areas the whole spectrum has a low intensity. As mentioned in the previous section, a Savitzky-Golay smoothing filter algorithm was used for noise removal.

Transmittance spectra were converted into absorption $(\log 1 / \mathrm{T})$ spectra. Fig 2 (B) shows average absorption spectra of the honey samples (50 pixels of each sample). The spectra illustrate typical spectral features and absorption bands similar to those reported for honey [51]. A clear absorption peak in the visible range at 400-425 $\mathrm{nm}$ is observed, which can be attributed to color variation of honey samples. Obviously, rapeseed honey despite its color similarity with lime and acacia honeys (white) shows a fairly different spectral pattern. Lighter honeys (except for rapeseed) have lower absorption in the visible range while in the NIR region the behavior is difficult to explain. It is especially the NIR range that is very important for discrimination between different floral origins.

\subsection{Dimensionality reduction by PCA}

High dimensional data in hyperspectral imaging should be reduced to remove the redundant data and represent the data distribution efficiently. Feature reduction can be achieved by selecting the more relevant subset of features or by transforming the data into a new set of axes in which differentiability is higher than in any subset of the original data [48].

Principal components analysis (PCA) is an eminent method for feature reduction which transforms the original independent variables into new axes [52]. In this study, PCA was carried out on all spectra. Loading plots of the first two components are given in Fig3 (A). The PC-1 loading plot does not emphasize specific wavelengths as all wavelengths have 
approximately equal weights in the NIR region. PC-2 shows that the highest loadings (eigenvectors) are found around $425 \mathrm{~nm}$ in the VIS region. This indicates that color, as the leading factor in honey, contributes to the discrimination of the floral origin. However, it would not be effective in differentiating honey samples having the same color which is confirmed by the literature [27]. The score plot of the first two PCs is depicted in Figure 3 (B). As it can be seen, the accumulative reliabilities of the first two PCs explain $99 \%$ of the total variance of spectra. It shows good separation of honey groups including acacia, buckwheat, rapeseed, and heather. Separation along PC1 appears to be more pronounced, as compared to separation along PC2 which concentrates on sample color as a differentiation factor. However, there is overlap between background-acacia and heather honey pixels. Some researchers indicated that in many cases PCA has minimal impact on detecting the statistical value for a target that is spectrally similar to the background against which it is sought [52]. Therefore, to achieve good separation, classification methods were applied as discussed below. The classification methods used in this study included SVM, RBF network and RF. It must be noted that the background of each spectral image was considered as a separate class.

\subsection{Prediction results}

Fig 4 illustrates the prediction performance of the three mentioned classifiers. This figure compares the precision of classifiers for feature vectors of the total spectrum as well as the reduced spectra of honey samples using PCA. Evidently, classification using the first two PCs or total spectrum as input features provides good classifier performance as precision is higher than $90 \%$ for all the three classifiers. The, RBF network provides the best discrimination between different types of honey as it achieved the highest precision value of $94 \%$ while it took $0.54 \mathrm{~s}$ to build the model. Performance evaluation of classifiers using total wavelengths as input feature vector did not show a significant change in the classifier 
performance and even led to higher computational time $(0.69 \mathrm{~s})$. Without dimensionality reduction, the SVM classifier shows the highest precision value (93\%).

Other classifiers including SVM and RBF network also have acceptable performance with negligible difference between them. Other performance parameters are presented in Table 1. Results show that RF and RBF classifiers achieved ROC values of $99 \%$ and $98 \%$, respectively for total spectrum and $2 \mathrm{Pcs}$ as classifier inputs. The other parameters including accuracy and recall ingeminate the near perfect performance for both mentioned classifiers. Results show the PCA analysis efficiency in reducing computational cost; so the RBF classifier with 2 PCs as input vector is the best performing classifier in both aspects of performance and computational time. In conclusion, results show the capability of hyperspectral imaging in achieving good discrimination between different types of honey based on floral origin. Although acacia and lime samples cannot be visually differentiated due to having the same color the current samples can be discriminated perfectly using hyperspectral imaging. The same holds for rapeseed and heather honeys. Minimal spectral information in the NIR region is responsible for these good results and the inclusion of spectral information from the NIR region was essential to obtain the most accurate honey classification. It is noted that all the information from the VIS-NIR radiation is involved in determining the model ability for honey floral origin detection, not just a specific region of the spectrum. The spectrum contains information about the entire composition of honey not just a particular compound.

Therefore, hyperspectral imaging proved to be beneficial in accomplishing an imaging discrimination system for honey floral origin detection. Since honey color changes over time, further studies are needed to determine the efficiency of NIR imaging during storage time.

\subsection{Hyperspectral mapping and misclassification investigation}


The result of classification can be transferred to the image to recognize its class (classifier mapping). Because of the good performance of the RBF network classifier for pixel-based classification of hyperspectral images, the trained RBF network classifier was applied to the PC-images (the first two PCs resulting from PCA analysis were applied for generating PC score images) of the 20 honey samples that were kept out for classifier testing. The resulting images were presented in colors where each color corresponds to a different type of honey, while the white color is used for the background. Pixels of each class (encountered in each sample) were counted to determine a majority "voting number" considered as the class of honey sample. The classifier was mapped into the images and the resulting images are shown in Fig 5. Vividly, from the image, the outer sides of microtiter for all types of honey are misclassified as rapeseed honey pixels in the image. Because of the curved surface of the samples at the outer edge, light transmittance through the samples was reduced. The resulting images also showed that one acacia sample has been classified incorrectly as rapeseed honey. In addition to this, one lime sample was classified as acacia honey. However, all other samples of heather, buckwheat, and rapeseed honeys were classified correctly.

\section{Conclusions}

This study was carried out to investigate the capabilities of advanced classifiers in conjunction with hyperspectral imaging for classification of various types of honey based on floral origin. Since hyperspectral imaging is a combination of machine vision and spectroscopy, it combines the advantages of both methods. Classification results showed that hyperspectral imaging could provide a robust method for discriminating different types of honey. Although, commercial honey is rather a homogenous product but in some cases crystallization will occur during the storage time and in the case of using NIR spectroscopy, sample preparation will be needed. The main advantage of hyperspectral imaging is that it can be operated on pixels anywhere in the sample. Classification results can be mapped on 
the acquired images, showing detailed spatial characteristics of the samples. Use of this spatial information, makes it possible to cope with impurities such as bubbles, sugar crystals and any contaminants. By using a simple "majority voting" mechanism, classification accuracy can be improved significantly. This method is fast and non-destructive in comparison with the traditional methods. Moreover, it provides the capability of accurate visualization of different classes of honey which is not possible with traditional methods. It alleviates the difficulties involved in other methods requiring sample preparation to remove bubbles, sugar crystals, and impurities found in natural honey. Since the efficiency of using visible imaging for measuring honey color and nutrient content has been indicated in the literature, the possibility of applying hyperspectral imaging for characterization of different aspects of honey (other than floral origin) in the future is promising.

\section{Acknowledgement}

The research work reported here has been supported by Tarbiat Modares University (grant No.82/120346) and the Polish National Science Centre, granted under the decision DEC2014/15/N/NZ9/04058. The authors gratefully acknowledge the financial support provided by the two institutions.

\section{References}

[1] V. Kaškonienè, P. R. Venskutonis, Floral Markers in Honey of Various Botanical and Geographic Origins: A Review, Compr. Rev. Food Sci. Food Saf. 9 (2010) 620-634.

[2] I. Escriche, M. Kadar, E. Domenech, and L. Gil-Sánchez, A potentiometric electronic tongue for the discrimination of honey according to the botanical origin . Comparison with traditional methodologies: Physicochemical parameters and volatile profile, J. Food. Eng. 109 (2012) 449-456. 
[3] P.C. Molan, The limitations of the methods of identifying the floral source of honeys, Bee World, 79 (1998) 59-68.

[4] K. Ruoff, Authentication of the botanical origin of honey. Doctor of science dissertation. University of Helsinki (2006).

[5] J.M. Stephens, R.C. Schlothauer, B.D. Morris , D. Yang, L. Fearnley, D.R. Greenwood, K. M. Loomes, Phenolic compounds and methylglyoxal in some New Zealand manuka and kanuka honeys, Food Chem. 120 (2010) 78-86.

[6] I.S. Arvanitoyannis, C. Chalhoub, P. Gotsiou, N. Lydakis-Simantiris, P. Kefalas, Novel quality control methods in conjunction with chemometrics (multivariate analysis) for detecting honey authenticity, Crit Rev Food Sci Nutr. 45 (2005) 193-203.

[7] A. Terrab, A.F. Recamales, M.L. Gonzalez-Miret, F.J. Heredia. Contribution to the study of avocado honeys by their mineral contents using inductively coupled plasma optical emission spectrometry, Food Chem. 92 (2005) 305-309.

[8] S. Bogdanov, K. Ruoff, L. Persano Oddo, Physico-chemical methods for the characterisation of unifloral honeys: a review. Apidologie. 35 (2004) 4-17.

[9] L. Persano Oddo, R. Piro, Main European unifloral honeys: descriptive sheets. Apidologie. 35 (2004) 38-81.

[10] O.M. Herńandez, J.M.G. Fraga, A.I. Jiḿenez, F. Jiḿenez, J. J. Arias, Characterization of honey from the Canary Islands: determination of the mineral content by atomic absorption spectrophotometry, Food Chem. 93 (2005) 449-58.

[11] P. Andrade, F. Ferreres, M.T. Amaral, Analysis of honey phenolic acids by HPLC, its application to honey botanical characterization, J Liq Chromatogr Relat Technol. 20 (1997) 2281-2288. 
[12] E. Alissandrakis, P.A. Tarantilis, P.C. Harizanis, M. Polissiou, Aroma investigation of unifloral Greek citrus honey using solid-phase microextraction coupled to gas chromatographic-mass spectrometric analysis. Food Chem. 100 (2007) 396-404.

[13] K.A. Aliferis, P.A. Tarantilis, P.C. Harizanis, E. Alissandrakis, Botanical discrimination and classification of honey samples applying gas chromatography/mass spectrometry fingerprinting of headspace volatile compounds. Food Chem. 121 (2010) 856-62.

[14] G. Beretta, E. Caneva, R.M. Facino, Kynurenic acid in honey from arboreal plants: MS and NMR evidence. Planta Medica. 73 (2007) $1592-5$.

[15] I. Rezić, A.J.M. Horvat, S. Babić, M. Kaštelan-Macan. Determination of pesticides in honey by ultrasonic solvent extraction and thin-layer chromatography, Ultrason. Sonochem. 12 (2005) $477-81$.

[16] M.J. Nozal, J.L. Bernal, L. Toribio, M. Alamo, J.C. Diego, J. Tapia. The use of carbohydrate profiles and chemometrics in the characterization of natural honeys of identical geographical origin. J. Agric. Food Chem. 53 (2005) 3095-100.

[17] J.A. Donarski, S.A. Jones, M. Harrison, M. Driffield, A.J. Charlton, Identification of botanical biomarkers found in Corsican honey. Food. Chem. 118 (2010), 987-994.

[18] C.I.G. Tuberoso, E. Bifulco, P. Caboni, F. Cottiglia, P. Cabras, I. Floris, Floral markers of strawberry-tree (Arbutus unedo L.) honey. J Agric Food Chem. 58 (2010) 384-389.

[19] M.M. Paradkar, J. Irudayaraj, Discrimination and classification of beet and cane inverts in honey by FT-Raman spectroscopy. Food Chem. 76 (2002) 231-239.

[20] E. Etzold, B. Lichtenberg-Kraag, Determination of the botanical origin of honey by Fourier-transformed infrared spectroscopy: an approach for routine analysis. Eur Food Res Technol. 227 (2008) 579-86. 
[21] K. Ruoff, W. Luginbühl, R. Künzli, M.T. Iglesias, S. Bogdanov, J.O. Bosset, R. Amadò. Authentication of the botanical and geographical origin of honey by mid-infrared spectroscopy, J. Agric. Food Chem., 54 (2006) 6873-688.

[22] E. Corbella, D. Cozzolino, The use of visible and near infrared spectroscopy to classify the floral origin of honey samples produced in Uruguay, J. Near Infrared Spectrosc. 68 (2005) 63-68.

[23] T. Woodcock, G. Downey, J.D. Kelly, C. O’Donnell, Geographical classification of honey samples by near-infrared spectroscopy: a feasibility study. J Agric Food Chem. 55 (2007) 9128-9134.

[24] L. Chen, J. Wang, Z. Ye, J. Zhao, X. Xue, Y. Vander Heyden, Q. Sun, Classification of Chinese honeys according to their floral origin by near infrared spectroscopy. Food Chem. 135 (2012) 338-342.

[25] S. Shafiee, S. Minaei, N. Moghaddam-Charkari, M. Ghasemi-Varnamkhasti, M. Barzegar, Potential application of machine vision to honey characterization. Trends Food Sci Tech. 30 (2013) 174-177.

[26] S. Shafiee, S. Minaei, N. Moghaddam-Charkari, M. Barzegar, Honey characterization using computer vision system and artificial neural networks. Food Chem. 159 (2014) 143150.

[27] S. Shafiee. Development of an intelligent System for Measurement of Some Physicochemical Attributes and Honey Adulteration Detection using machine vision and NIR Spectroscopy. Doctor of science dissertation, TarbiatModares University (2015).

[28] D. Wu, D.W. Sun, Advanced applications of hyperspectral imaging technology for food quality and safety analysis and assessment: A review — Part II: Applications. Innov. Food Sci. Emerg. 19 (2013) 15-28. 
[29] S. Shafiee, G. Polder, S. Minaei, N. Moghadam-Charkai, P.M. Kuś, V. Ruth, Detection of Honey Adulteration using Hyperspectral Imaging. IFAC-Papers. 49-16 (2016) 311314.

[30] M.E. Schaepman, Spectrodirectional remote sensing: From pixels to processes. Int J Appl Earth Obs Geoinf. 9 (2007) 204-223.

[31] G. Elmasry, M. Kamruzzaman, D.W. Sun, P. Allen, Principles and applications of hyperspectral imaging in quality evaluation of agro-food products: a review. Crit Rev Food Sci. 52 (2012) 999-1023.

[32] G. Shaw, D. Manolakis, Signal processing for hyperspectral image exploitation. IEEE Signal Processing Magazine. 19 (2002) 12-16.

[33] G.K. Naganathan, I.M. Grimes, J. Subbiah, C.R. Calkins, A. Samal, G.E. Meyer, Visible/near-infrared hyperspectral imaging for beef tenderness prediction. Comput Electron Agric. 64 (2008) 225-233.

[34] J. Qiao, M.O. Ngadi, N.Wang, C. Gariepy, S.O. Prasher, Pork quality and marbling level assessment using a hyperspectral imaging system. J Food Eng. 83 (2007) 10-16.

[35] V.H. Segtnan, M. Høy, F. Lundby, B. Narum, J.P. Wold. Fat distributional analysis in salmon fillets using non-contact near infrared interactance imaging, a sampling and calibration strategy. J Near Infrared Spectrosc. 17 (2009) 247-253.

[36] D. Wu, D.W. Sun, Y. He, Application of long-wave near infrared hyperspectral imaging for measurement of color distribution in salmon fillet. Innov. Food Sci. Emerg. Technol. 16(2012), 361-372.

[37] H.J. He, D. Wu, D.W. Sun. Non-destructive and rapid analysis of moisture distribution in farmed Atlantic salmon (Salmosalar) fillets using visible and near-infrared hyperspectral imaging. Innov. Food Sci. Emerg. Technol. 18 (2013) 237-245. 
[38] J. Li, X. Rao, Y. Ying, Development of algorithms for detecting citrus canker based on hyperspectral reflectance imaging, J Sci Food Agric. 92 (2012) 125-34.

[39] G.A. Leiva-Valenzuela, R. Lu, J.M. Aguilera, Assessment of internal quality of blueberries using hyperspectral transmittance and reflectance images with whole spectra or selected wavelengths, Innov. Food Sci. Emerg. Technol. 24 (2014) 2-13.

[40] G. Polder, G. W. A. M. Van Der Heijden, I.T. Young. Spectral image análysis for measuring ripeness of tomatoes. T ASABE. 45 (2002) 1155-1161.

[41] C. Mo, G. Kim, M. S. Kim, J. Lim, S.H. Lee, H. Lee, B. Cho. Discrimination methods for biological contaminants in fresh-cut lettuce based on VNIR and NIR hyperspectral imaging. Infrared Phys. Technol. (2017). https://doi.org/10.1016/j.infrared.2017.05.003

[42] X. Fu, M.S. Kim, K. Chao, J. Qin, J. Lim, H. Lee, A. Garrido-Varo, D. Pérez-Marín, Y. Ying, Detection of melamine in milk powders based on NIR hyperspectral imaging and spectral similarity analyses, J. Food Eng. 124 (2014) 97-104.

[43] J. Louveaux; A. Maurizio; G. Vorwohl. Methods of Melissopalynology. Bee World 59 (1978) 139-157.

[44] W. Von Der Ohe, L. Persano Oddo, M.L. Piana, M. Morlot M and P. Martin. Harmonised methods of melissopalynology. Apidologie. 35 (2004) 18-25.

[45] G. Polder, G. W. A. M. Van Der Heijden, L.C.P. Keizer, I.T. Young. Calibration and characterization of imaging spectrographs. J Near Infrared Spectrosc. 11 (2003) 193-210.

[46] M. Akbaripour-elahabad, J. Zahiri, R. Forest. rpiCOOL : A Tool for In Silico RNA Protein Interaction Detection Using Random Forest Reference. J. Theor. Biol. 402 (2016) $1-8$. 
[47] X. Zhu, S. Li, Y. Shan, Z. Zhang, G. Li, D. Su, F. Liu. Detection of adulterants such as sweeteners materials in honey using near-infrared spectroscopy and chemometrics. J. Food Eng. 101 (2010) 92-97.

[48] L. Jiang, B. Zhu, Y. Tao, Hyperspectral Imaging for Food Quality Analysis and Control. Elsevier, (2010)79-98.

[49] A.P. Manjunatha, H.S. Sheshadri, M.N. Giriprasad. Performance analysis of Neural Network based classification technique for Mammogram Images. Int J Applied Eng Res. 11 (2016) 7550-7554.

[50] J. Zahiri, J.H. Bozorgmehr, A. Masoudi-Nejad. Computational Prediction of Protein Protein Interaction Networks: Algorithms and Resources. Curr. Genomics. 14 (2013) 397414.

[51] E. Corbella, D. Cozzolino. The use of visible and near infrared spectroscopy to classify the floral origin of honey samples produced in Uruguay, J. Near Infrared Spectrosc. 68 (2005) 63-68.

[52] I. Esteban-Diez, J.M. Gonzalez-Saiz, C. Pizarro. An evaluation of orthogonal signal correction methods for the characterisation of arabica and robusta coffee varieties by NIRS. Analytical Chimica Acta. 514 (2004) 57-67.

[53] M. D. Farrell, S. Member, R. M. Mersereau. On the Impact of PCA Dimension Reduction for Hyperspectral Detection of Difficult Targets, IEEE. 2 (2005) 192-195. 


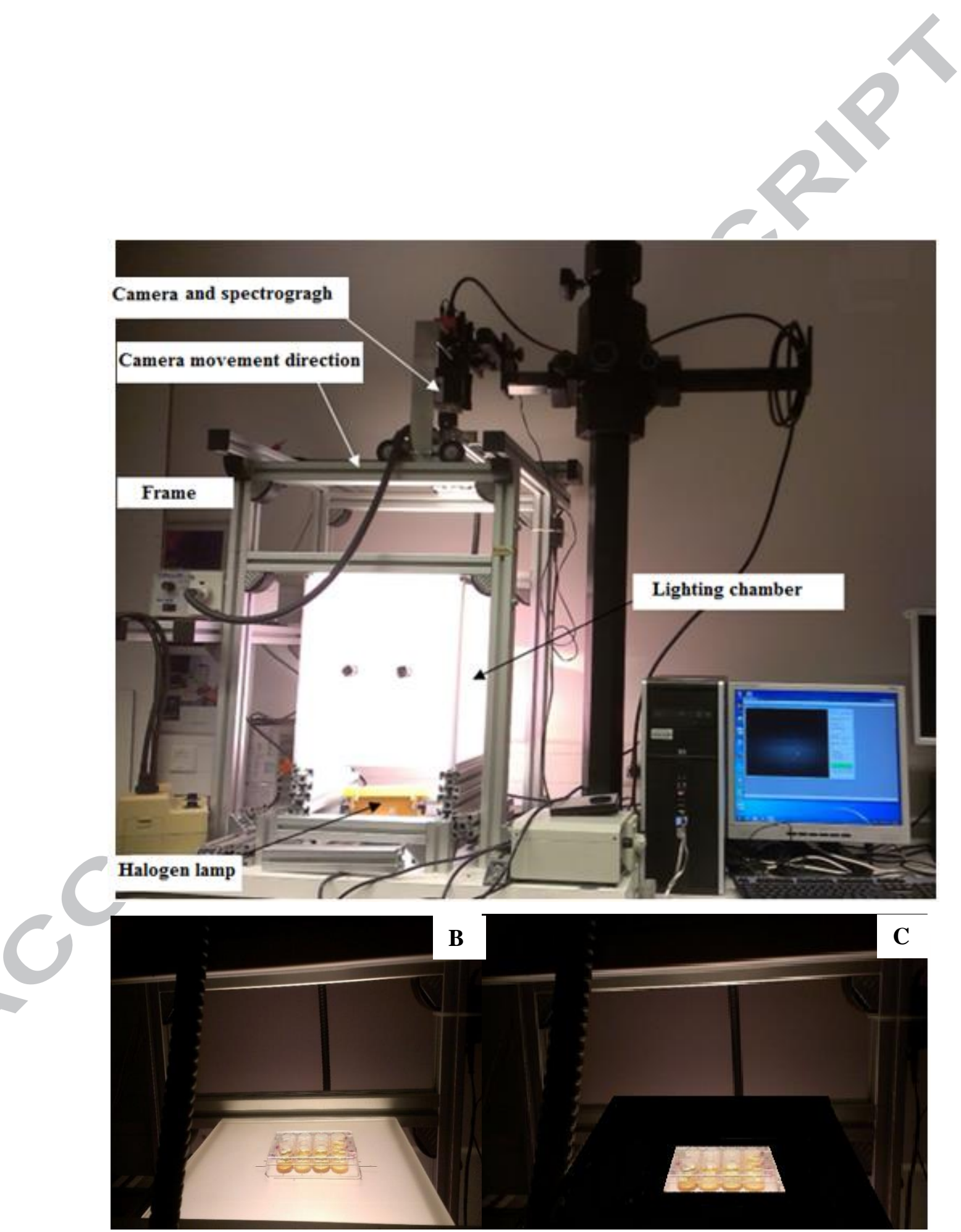

Figure 1. Hyperspectral imaging system overview (A), Sample placement (B), Black sheets for light covering (C) 




(A)

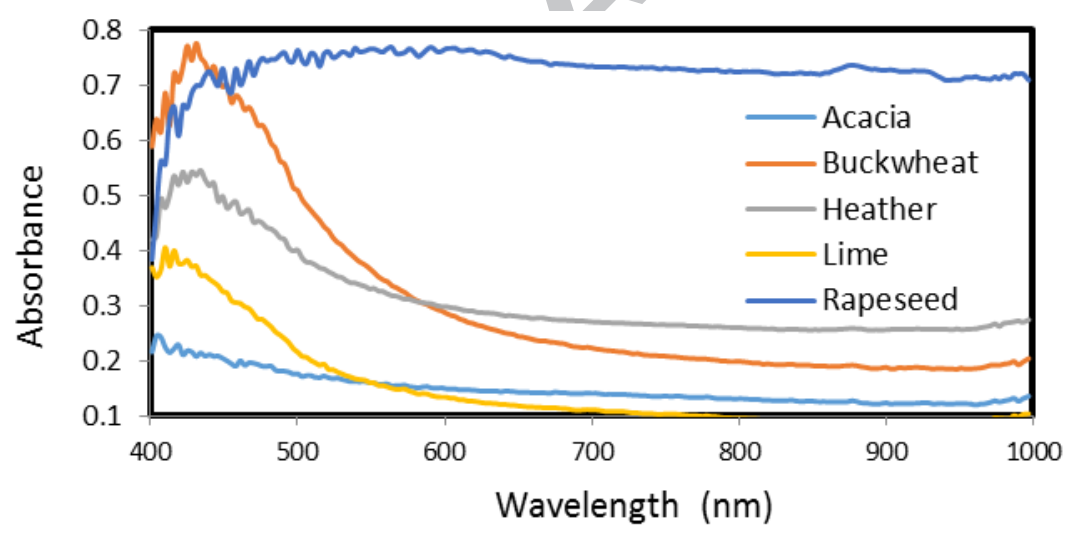

(B)

Figure 2. Sample of the selected image pixels and their related raw spectra for honey (blue) and background (red) (A), Average absorbance for the five honey floral origin(B) 

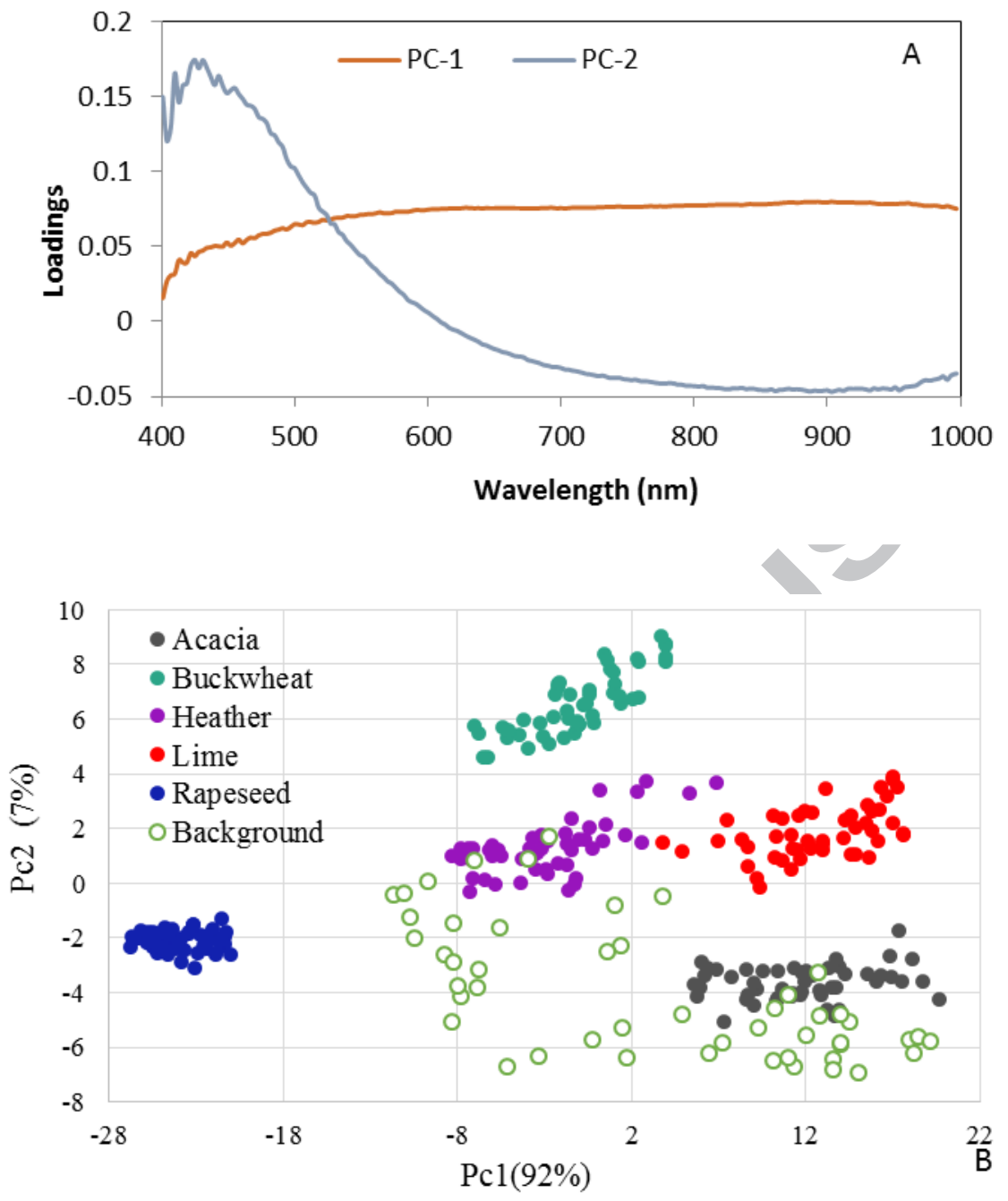

Figure 3. The loadings plot of PC1 and PC2 (A), Score plot of PC1 and PC2 (B). 


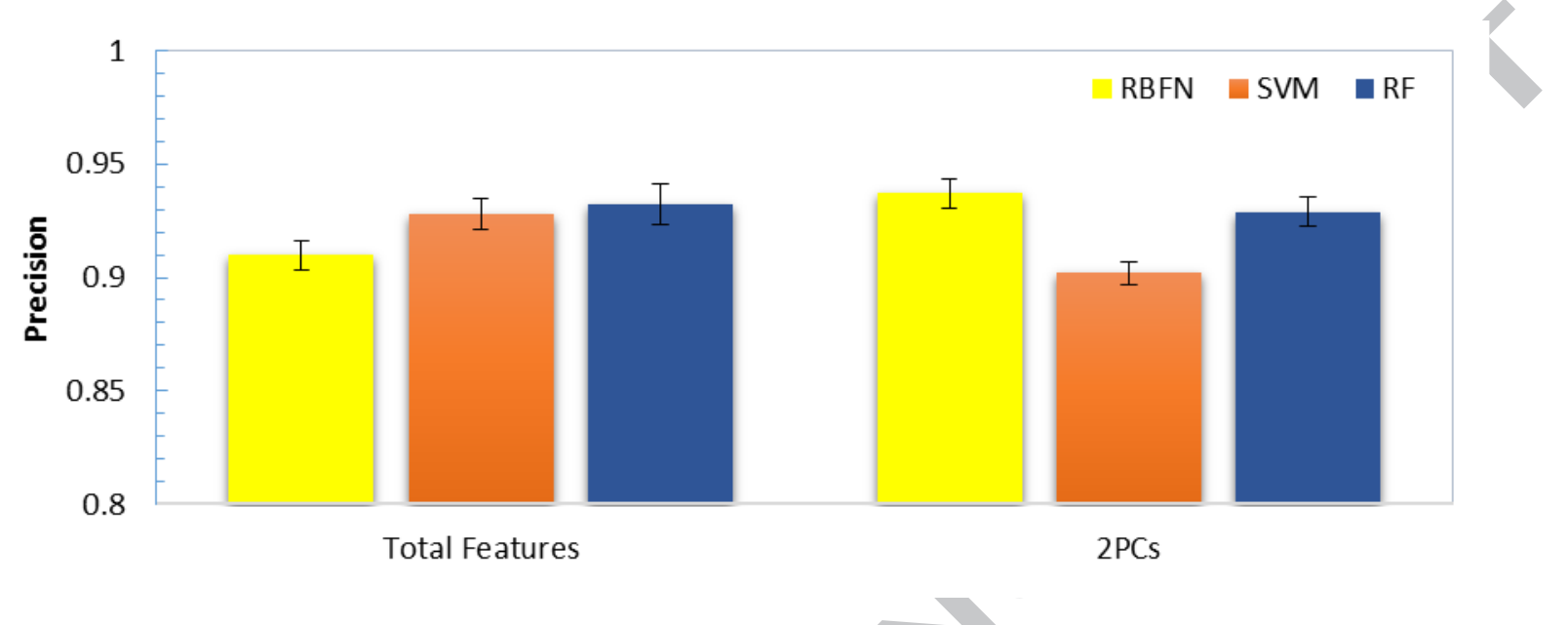

Figure 4. Comparison of classifiers precision 


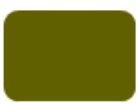

acacia

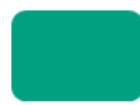

buckwheat

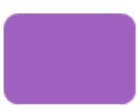

heather

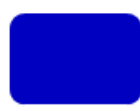

rapeseed

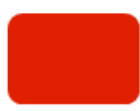

Lime



background

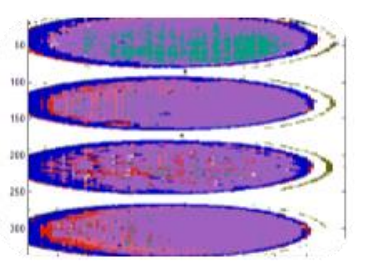

heather

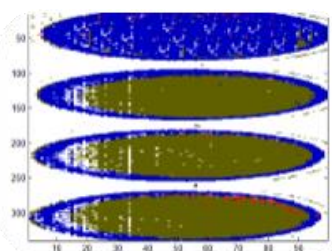

acacia



Lime



Figure 5- Honey class images as grouped by the RBF network 
Table 1. Performance measure details for three classifiers (mean \pm standard deviation)

\begin{tabular}{ccccc}
\hline Classifier input & & RBF & SVM & RF \\
\hline \multirow{3}{*}{ Total spectrum } & ROC & $0.97 \pm 0.012$ & $0.98 \pm 0.001$ & $\mathbf{0 . 9 9} \pm \mathbf{0 . 0 0 4}$ \\
& Recall & $0.90 \pm 0.008$ & $0.90 \pm 0.014$ & $\mathbf{0 . 9 2} \pm \mathbf{0 . 0 1 5}$ \\
& ACC $(\%)$ & $90.97 \pm 0.006$ & $90.52 \pm 1.5$ & $\mathbf{0 . 9 2} \pm \mathbf{1 . 5 7}$ \\
\hline \multirow{2}{*}{ 2PC } & ROC & $\mathbf{0 . 9 8} \pm \mathbf{0 . 0 3 9}$ & $0.961 \pm 0.093$ & $0.965 \pm 0.032$ \\
& Recall & $\mathbf{0 . 9 2} \pm \mathbf{0 . 0 0 6}$ & $0.844 \pm 0.069$ & $0.889 \pm 0.099$ \\
& ACC $(\%)$ & $\mathbf{9 2 . 2 2} \pm \mathbf{0 . 5 5}$ & $84.4 \pm 1.28$ & $88.89 \pm 1.26$ \\
\hline
\end{tabular}

